\title{
Connectivity in a UAV Multi-static Radar Network
}

\author{
David W. Casbeer* and A. Lee Swindlehurst ${ }^{\dagger}$ and Randal Beard ${ }^{\ddagger}$ \\ Department of Electrical and Computer Engineering \\ Brigham Young University, Provo, UT
}

\begin{abstract}
This paper describes a multi-static radar network composed of multiple unmanned air vehicles (UAVs). Time-delay and Doppler measurements taken by the UAV team are passed to a centralized processor to determine optimal heading commands for the UAV team to reduce tracking error. However, due to power and communication constraints on the UAVs, the optimal heading could cause individual UAVs or perhaps the entire network to become disconnected from the base station. In this paper, we ensure that the UAV team remains connected to the base by adding a connectivity constraint to the optimization criterion. This constraint is a modification to the "geometric connectivity robustness" metric proposed recently by Spanos and allows for a balance between the sensor network's performance and connectivity, while ensuring the appropriate connections for necessary information flow.
\end{abstract}

\section{Introduction}

In this paper, we consider a multi-static radar tracking scenario where the radar receivers are mounted on individual unmanned air vehicles (UAVs). The defining aspect of this problem is the added measure of freedom inherent with the mobility and autonomy of the UAV platforms. It has been shown that the team's mobility can dramatically improve tracking performance compared with a static radar array receiver. ${ }^{1}$ This previous work relied on the assumption that all UAVs were connected via a communications link to a central processor or base station. The base uses the link to collect time-delay and Doppler measurements, along with the UAVs' location and velocity. Using this data, the base then determines optimal headings for the UAVs in order to improve tracking performance, and sends this information back to the UAVs via the link.

The centralized solution described above made no provision to require that the UAVs, together with the base, formed a connected network allowing for information flow from the base to all UAVs and vice versa. In this paper we address this networking issue by adding a connectivity constraint to the tracking performance criterion, ensuring that each UAV is connected to the base station either directly or by a multi-hop relay through other UAV team members.

For general joint-task control problems, the use of autonomous agents adds significant complexity. In order for the task to be carried out effectively, the agents must typically be aware of the actions of their neighbors. Complete connections between all agents is not necessary, but in order to achieve acceptable performance, a certain level of network connectivity must be maintained. ${ }^{2,3}$ Recently, graph theoretic $^{4,5}$ as well as ad-hoc ${ }^{6-8}$ approaches have been taken to constrain agents' behavior so that network connections remain intact during the joint operations.

A situation related to the tracking scenario described above is the coverage/connectivity problem, in which multiple sensors are required to cover a region of interest while remaining connected for communicating measurements. One approach to solving this problem is intelligently selecting the activation or sleep modes of the sensing nodes. ${ }^{9-11}$ For situations involving grid searches, the deployment and movement of multiple agents can be constrained to certify connectivity. ${ }^{12}$ Another approach to the autonomous joint radar tracking problem has been to ensure the formation of small groups of radar receivers, ${ }^{13}$ where each group is comprised of enough agents to solve for the target states from their measurements without singularity problems. In

*PhD Candidate, casbeer@byu.edu

$\dagger$ Professor, swindle@ee.byu.edu

¥Associate Professor, beard@ee.byu.edu 
essence, each individual group tracks the target itself, and when the network configuration allows it, the current group estimates are shared to enhance estimation and tracking.

The work presented in this paper closely follows that of Refs. 7,8, where a local "geometric connectivity robustness" metric is defined. This metric is local in the sense that one node is only dependent on the nodes it needs to share information with and any associated relay nodes. Here we differ in that we use prior knowledge concerning network information flow and the unconstrained optimal trajectories to modify Spanos' connectivity robustness metric. This adaptation results in each UAV being dependent on only the adjacent relay node between itself and the base station. The benefit produced by this change is that more reliable target data is acquired through the UAV team's sensor geometry.

The arrangement of the paper is as follows. The multi-static UAV radar network with unconstrained heading control will be discussed in Section III Section III then discusses the concept of geometric connectivity robustness, followed by our modification in Section [IV] In Section [V] simulations are presented showing the performance trade-off between our modification and the original connectivity robustness metric. Some conclusions and areas for future work are discussed in Section VI

\section{Multi-Static UAV Radar Network}

The sensor network problem addressed in this paper is a multi-static radar tracking scenario depicted in Figure 11 where an airborne target is illuminated with a signal transmitted from some known location. The reflection of this signal from the target is received by multiple UAVs, who extract both time-delay and Doppler information. These measurements are then collected by a base station for use in estimating the target's position and velocity. In this section we briefly present a model for this problem; more detail can be found in Refs. 1,14.

We will denote the position of the transmitter and target as $\mathbf{q}_{b}=\left[x_{x}, y_{x}\right]^{T}$ and $\mathbf{q}_{t}=\left[x_{t}, y_{t}\right]^{T}$, respectively. The position of each UAV and the base station in the $N$ agent team will be denoted by $\mathbf{q}_{i}=\left[x_{i}, y_{i}\right]^{T}, i=$ $0, \ldots, N$, where the base station is indicated by the index $i=0$.

\section{A. Measurements \& Tracking}

The time-delay and Doppler measurements taken at the UAVs are related to the target parameters via the following equations:

$$
\begin{aligned}
\tau_{i} & =\frac{1}{c}\left(d_{b t}+d_{t i}\right) \\
\omega_{i} & =-\omega_{c}\left(\frac{\partial d_{b t}}{\partial t}+\frac{\partial d_{t i}}{\partial t}\right)
\end{aligned}
$$

where $c$ is the speed of light, $\omega_{c}$ is the radar carrier frequency, $d_{b t}=\left\|\mathbf{q}_{b}-\mathbf{q}_{t}\right\|$ represents the distance from the transmitter to the target, and $d_{t i}=\left\|\mathbf{q}_{t}-\mathbf{q}_{i}\right\|$ represents the distance from the target to UAV $i$. Assuming knowledge of the transmitter and UAV locations, the time-delay and Doppler measurements depend only on the unknown parameters of the target. These measurements can then

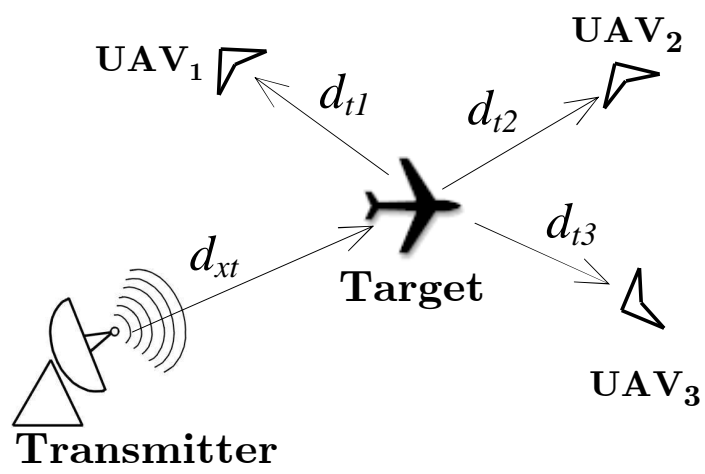

Figure 1. Transmitter emits signal that bounces off the target and is received by the UAVs. be used at the base station in an Extended Kalman Filter (EKF) framework for tracking the target.

To describe the operation of the EKF, we first stack the position and velocity of the target at time $k$ into the vector

$$
\mathbf{s}_{t}=\left[x_{t}, y_{t}, v_{t} \cos \psi_{t}, v_{t} \sin \psi_{t}\right]^{T} .
$$

For our purposes, the key information provided by the EKF is the covariance of the linearized target state parameter estimate $\hat{\mathbf{s}}_{t}$. This covariance matrix is propagated in time according to the following recursion:

$$
\begin{aligned}
\mathbf{P}_{k} & =E\left[\left(\mathbf{s}_{t}-\hat{\mathbf{s}}_{t}\right)\left(\mathbf{s}_{t}-\hat{\mathbf{s}}_{t}\right)^{T}\right]=\left(\mathbf{I}-\mathbf{K}_{k} \mathbf{H}_{k}\right)\left(\mathbf{A} \mathbf{P}_{k-1} \mathbf{A}^{T}+\mathbf{Q}_{k}\right) \\
\mathbf{K}_{k} & =\mathbf{P}_{k-1} \mathbf{H}_{k}^{T}\left(\mathbf{H}_{k} \mathbf{P}_{k-1} \mathbf{H}_{k}^{T}+\mathbf{R}_{k}\right)^{-1}
\end{aligned}
$$


where $k$ is the current time step, $\mathbf{K}_{k}$ is the Kalman gain, $\mathbf{A}$ is the linearized state transition matrix describing the target's motion, $\mathbf{R}_{k}, \mathbf{Q}_{k}$ are respectively the measurement and process noise covariance matrices, and $\mathbf{H}_{k}$ is a linearization of the aggregate UAV measurements,

$$
\mathbf{h}_{k}=\left[\tau_{1} \ldots \tau_{N}, \omega_{1} \ldots \omega_{N}\right]^{T}
$$

about the estimated target state, $\hat{\mathbf{s}}_{t}$. After expressing the $j^{\text {th }}$ component of the target's parameter $\mathbf{s}_{t}$ as $s_{t}^{j}$ and the $i^{t h}$ component of the collective measurement $\mathbf{h}_{k}$ as $h_{k}^{i}$, the $(i, j)^{t h}$ element of $\mathbf{H}_{k}$ can be written as

$$
\left(\mathbf{H}_{k}\right)_{j}^{i}=\left.\frac{\partial h_{k}^{i}}{\partial s_{t}^{j}}\right|_{\hat{\mathbf{s}}_{t}}
$$

\section{B. Optimal Heading \& Example Trajectory}

As seen from Equations (15), the position and velocity of the UAV team members influences the propagation of $\mathbf{P}_{k}$ through the gradient of the measurement model, $\mathbf{H}_{k}$. Our desire is to improve tracking by exploiting our control over the agents. Each UAV is assumed to be equipped with an autopilot tuned for first order response to heading commands with a constant altitude hold. ${ }^{15}$ Writing the position of UAV $i$ in terms of its heading after a short time step $\Delta t$, we have

$$
\mathbf{q}_{i, k+1} \approx \mathbf{q}_{i, k}+\Delta t \sqrt{v_{i, x}^{2}+v_{i, y}^{2}}\left[\begin{array}{c}
\cos \psi_{i} \\
\sin \psi_{i}
\end{array}\right] .
$$

Using this motion model for each UAV, the base station minimizes the trace of the EKF error covariance at the next time step with respect to the UAV headings $\Psi=\left[\psi_{1} \ldots \psi_{N}\right]^{T}$ :

$$
\Psi_{\text {opt }, 1}=\underset{\Psi}{\arg \min } \operatorname{Tr}\left\{\mathbf{P}_{k+1}\right\} .
$$

This optimal heading is then downloaded to each UAV agent as a heading command.

A sample trajectory flown by two UAVs using this optimization criterion is shown in Figure 2 In this example the transmitter and base station are co-located at the origin. The motion ascribed to the UAV agents here is typical. They tend to follow the target's flight path while spreading slightly away to obtain a wider field-of-view. Since the quality of the time-delay and Doppler estimates depends on the SNR of the reflected signal, the UAVs generally tend to try and follow the target to maintain signal strength. Thus, when the target is moving away from the base, the UAVs will move away as well.

\section{Network Connectivity}

From the previous section, it is clear that each UAV agent must remain in contact with the base station in order for the measurements to be collected and the heading commands to be fed back. However, the resulting UAV trajectories tend to take them

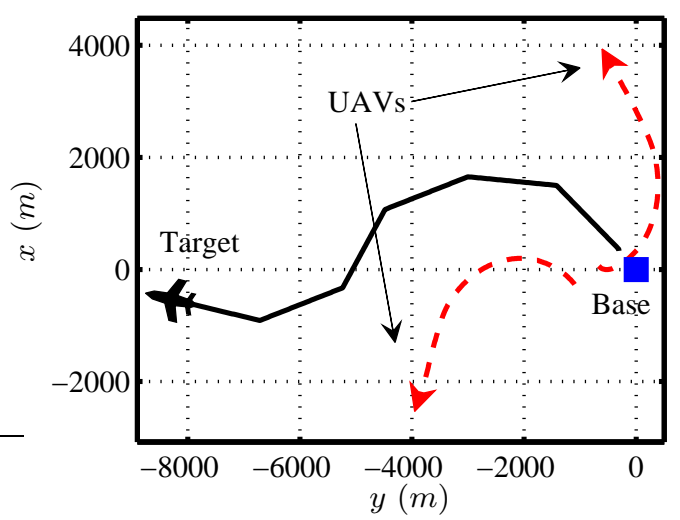

Figure 2. Two UAVs optimally tracking a target. The UAVs appear as red arrow tips and the target as a green airplane.

away from the base, a behavior that if left unchecked will soon put the UAVs out of range for reliable communications. This will especially be true for the UAV-to-base link, since small UAVs are often severely power constrained. To address this situation, in this section we describe a distributed networking metric that simultaneously balances the sensor network's performance with its need for connectivity.

Two undirected graphs that are used to describe a network include the communication network graph and the information flow graph. ${ }^{7,8}$ Following graph theoretic terminology, we use the term "node" to indicate elements of the communication network. In our application, these nodes comprise the base station and the 
$N$ UAV agents. The set of $N+1$ nodes will be denoted by $\mathbb{N}$. An edge $\varepsilon=\{i, j\} \in \mathbb{N}^{2}$ is a member of the communication network graph, $C=\left(V_{C}, E_{C}\right)$, if the communication link between nodes $i$ and $j$ is bidirectional. In mathematical notation this is written as

$$
\{i, j\} \in E_{C} \text { iff } \min \left\{r_{i}, r_{j}\right\}-d_{i j} \geq 0
$$

where $r_{i}$ is the communication range for $\mathrm{UAV}_{i}$ (i.e., UAV $i$ can communicate with any agents inside a circle of radius $r_{i}$ centered at $\mathbf{q}_{i}$ ), and $d_{i j}$ is the distance between $\mathrm{UAV}_{i}$ and $\mathrm{UAV}_{j}$. The communication neighborhood of node $i$ is then

$$
N_{C}(i)=\left\{j \in \mathbb{N}: \min \left\{r_{i}, r_{j}\right\}-d_{i j} \geq 0\right\}
$$

which is interpreted as "node $j$ is in the communication neighborhood of node $i\left(N_{C}(i)\right)$, if there is a bidirectional link between these two nodes."

The information flow graph, $I=\left(V_{I}, E_{I}\right)$, indicates which nodes need to share information. In this paper all information sharing is symmetric, thus a pair of nodes $\varepsilon_{I}=\{i, j\} \in \mathbb{N}^{2}$ is a member of the information flow graph's edge set, $E_{I}$, if and only if node $i$ requires information from node $j$ and vice versa. The information neighborhood, $N_{I}(i)$, associated with a given node $i$ is the set of nodes on which node $i$ relies.

The following path robustness ${ }^{7}$ metric quantifies the strength of the connection between nodes $i$ and $j$ :

$$
P(i, j, k)=\min \left\{\min \left\{r_{i}, r_{k}\right\}-d_{i k}, \min \left\{r_{j}, r_{k}\right\}-d_{j k}\right\} .
$$

This metric is only valid when the relay node $k$ is a member of the communication neighborhood of node $i$, otherwise $P(i, j, k)$ becomes negative. The metric can be extended to longer hop lengths, but a two hop scenario is sufficient in our case. The goodness of the network topology relative to the information flow, $I$, can be quantified using the geometric connectivity robustness: ${ }^{8}$

$$
R_{I}(i)=\min _{j \in N_{I}(i) \cup N_{C}(i)}\left[\frac{1}{2} \max _{k \in N_{C}(i)} P(i, j, k)\right] .
$$

For agent $i$, this metric determines the weakest link in the connections to all nodes in its information neighborhood. This weakest link can then be strengthened to improve communication (e.g., by increasing transmit power, adjusting node position, etc.). The assumption is that the metric will be locally continuous over small adjustments to the network topology.

\section{Adapting the Communication Neighborhood}

Connectivity robustness is a local metric indicating how well a node is connected to its information neighborhood. This metric will tend to cluster nodes in the same communication neighborhood, which can be seen in the minimization over the information and communication neighborhoods (e.g., Equation (10)). In the radar tracking scenario this clustering is undesirable because the agents will tend to group together and hence diminish tracking performance.

For a given problem, the information neighborhood for node $i$ is fixed. However, it might be possible to adapt the communication neighborhood to improve sensor performance at the cost of reducing the network connectivity. Rather than including all nodes within range, an agent could add and remove nodes within its communication neighborhood intelligently. To illustrate the possible gains, we introduce a link neighborhood as a specialized subset of the communication neighborhood. By adapting this subset, sensor performance can be improved. In order to illustrate the possible performace gains we will examine two subsets of the communication neighborhood, namely using the full communication neighborhood and a geometrically constrained subset of the communication neighborhood to be described in the next section.

\section{A. Link Neighborhood}

The information flow graph for this tracking problem is a rooted tree ${ }^{16}$ with $N+1$ nodes, where the root is the base station (see figure 3(a). Data travels from each node toward the center of the graph as well as from the center back to each node. Because of communication constraints, we know that each agent must be connected to the base, either directly or indirectly through a relaying neighbor UAV. Each agent then has a strong desire that the relay links to the base station be well connected. 


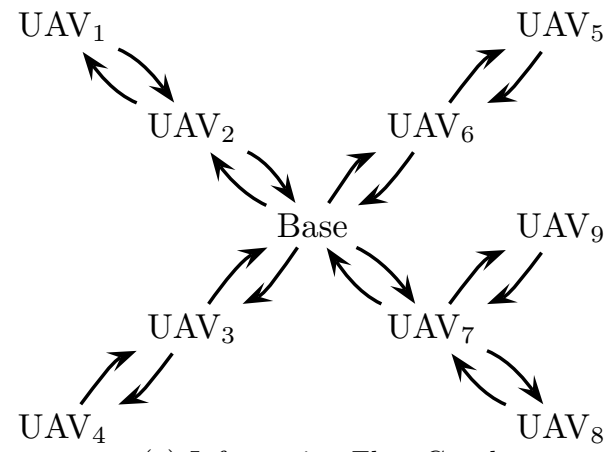

(a) Information Flow Graph

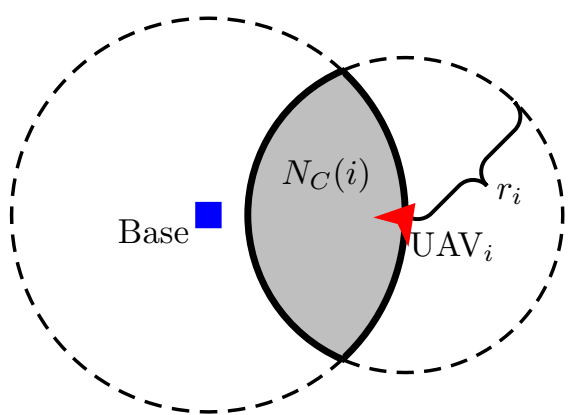

(b) Depiction of link neighborhood, where base is not within communication distance.

Figure 3. Depiction of the information flow graph and communication neighborhood.

The link neighborhood for agent $i, N_{L}(i)$, is defined as a subset of the communication neighborhood. This subset would presumably be defined to fit some performance criteria that would either constrain communication and/or sensor performance. Here, because we know the direction of the information flow, it seems appropriate for a node to only look at links that are in the direction of its information flow graph. A node farther away from the base station would most likely be an inappropriate connection to the base station. Therefore we take the link neighborhood to be

$$
N_{L}(i)=\left\{j \in N_{C}(i):\left\|\mathbf{q}_{i}-\mathbf{q}_{b}\right\|>\left\|\mathbf{q}_{j}-\mathbf{q}_{b}\right\|\right\} .
$$

All agents closer to the base station than UAV $i$ that are also within communication range of UAV $i$ are included in the link neighborhood, $N_{L}(i)$. This is graphically depicted in Fig. 3(b)] when the base station lies outside the communication range of the UAV. It can be seen here that defining the link neighborhood in this manner removes nodes from the communication neighborhood that will not help it relay information to its information neighborhood (i.e., agents that are in the opposite direction from the information flow). Each node must work to keep the links that help them relay data to their individual information neighborhoods.

In the case presented, the link neighborhood was taken as an ad-hoc geographic subset of communication neighborhood based on the information flow graph. Obviously, smarter choices could be made to choose this subset. Smarter parameters influencing the link neighborhood could include the desires and states of neighboring nodes such as: who is included in neighboring nodes' information neighborhood, their location and direction, and the number of nodes with whom they are currently communicating or relaying information.

\section{B. Modified connectivity robustness}

We modify the connectivity robustness measure by requiring an agent to look only at one hop edges connecting itself with members of its link neighborhood, rather that at multi-hop paths. The modified connectivity robustness measure is then

$$
\tilde{R}_{I}(i)=\max _{j \in N_{L}(i)} P(i, j)
$$

where $P(i, j)$ is the one edge path from node $i$ to $j$. If the link neighborhood is empty the UAVs will seek out the base station.

This metric only looks at the best connection in the link neighborhood, where the important characteristic of all nodes in the link neighborhood for UAV $i$ is that these nodes desire to communicate with the same information neighborhood as UAV $i$. This characteristic is inherent in the centralized tracking problem where all nodes desire to communicate with the base station. By taking only the best connection, this modified connectivity robustness metric is taking the best link in the communication neighborhood, which is essentially taking the smallest feasible subset of the communication neighborhood.

In a more complicated scenario with larger information neighborhoods, a UAV would need to define multiple link neighborhoods, one link neighborhood for every element of the information neighborhoods. Again, it is important that elements of node $i$ 's link neighborhood desire to communicate with the elements node 
$i$ 's information neighborhood. This would ensure that information flows toward members of the information neighborhood.

The modified connectivity robustness measure is better suited to our problem than the original, because each node itself focuses on what actions it must take to stay connected to its information neighborhood. This is because each UAV only depends on information from its neighbors rather than all agents on the paths connecting it to the base. However, additional information is needed in order to define link neighborhoods, namely an agent must know the the information neighborhoods of its neighbors. This metric is less constricting that the original, allowing the sensor network's geometry to better optimize for improved tracking performance. It allows long hop paths easily; more work is needed to restrict the number of relays allowed. More robust connectivity could be maintained by using multiple of the stronger links, rather than the best link alone; more work is needed to develop this tradeoff.

\section{Control Formulation}

The heading control algorithm with geometric connectivity robustness constraints can be formulated as

$$
\Psi_{o p t, 2}=\underset{\Psi}{\arg \min }\left\{\operatorname{Tr}\left\{\mathbf{P}_{k+1}\right\}+\sum_{i} \frac{\alpha}{\left|R_{I}(i)\right|}\right\} .
$$

The optimal heading solution is constrained by the current heading of each UAV and their individual dynamics. For the case where the link neighborhood is utilized, the geometric connectivity robustness, $R_{I}(i)$, in Equation (13) is replaced with the modified version, $\tilde{R}_{I}(i)$, given in Equation (12) yielding,

$$
\Psi_{o p t, 3}=\underset{\Psi}{\arg \min }\left\{\operatorname{Tr}\left\{\mathbf{P}_{k+1}\right\}+\sum_{i} \frac{\alpha}{\left|\tilde{R}_{I}(i)\right|}\right\} .
$$

\section{Results}

The three simulations presented are used to illustrate the improved tracking performance together with the network connectivity loss tradeoff. The simulations test the optimal one-step heading controller from Eq. (7) with no constraints, the heading controller with the connectivity robustness constraint of Eq. [13] and the heading controller with the modified connectivity robustness control formulation in Eq. (14), which trades off improved tracking performance for lower network connectivity. The performance of the sensor system is measured using the trace of the one-step ahead update covariance, ${ }^{1} \operatorname{Tr}\left\{\mathbf{P}_{k+1}\right\}$, while connectivity is measured using the second eigenvalue, $\lambda_{2}\left(L_{G}\right)$, of the network graph's Laplacian, $L_{G} .{ }^{3,16}$

The radar transmitter and base station are co-located and fixed at the origin. The coordinates in meters for the initial positions of the four UAVs are: $\mathrm{UAV}_{1},(1580,-855), \mathrm{UAV}_{2},(-995,1731), \mathrm{UAV}_{3},(-1476,1763)$, and $\mathrm{UAV}_{4},(807,1391)$. The UAVs travel with a constant velocity of $10 \mathrm{~m} / \mathrm{s}$. The target starts at $(392,1797)$ and is moving at $21 \mathrm{~m} / \mathrm{s}$. Its trajectory is identical for all three simulations.

In Figure 4 the trajectories for the three scenarios are shown. The maximum communication distance was set at $3.5 \mathrm{~km}$ for the two communication-constrained situations. The tracking and connectivity measures are plotted in Figure 5 The connectivity robustness constraint ensures that the edge connection between neighboring nodes remain fixed and this is reflected in the constant connectivity measure. The modified connectivity robustness method utilizing the link neighborhood as defined in Eq. (12) greatly improves the the tracking performance while ensuring the connectivity of the network, albeit at a lower level.

\section{Conclusion}

A modified connectivity robustness metric has been proposed that trades off improved tracking performance in a multi-static UAV network for network connectivity. Although connectivity is reduced, the criterion attempts to ensure that the network remains connected. The presented solution is centralized, in that it relies on the presence of a base station for computing and distributing heading commands to the UAVs. Research into the concept of a link neighborhood and the modified connectivity robustness metric in a decentralized system where the information flow graph is of larger dimension is still needed.

Smart adaptation of the link neighborhood in a decentralized situation could improve the sensors' performance while simultaneously guaranteeing a certain degree of connectivity in the network. Ideas for such 
improvements could use the neighbors' communication preferences such as their information neighborhood, as well as the neighbors' preference for optimal sensing coverage. Other networking metrics such as capacity or transmission rate could also be used to balance coverage and communication needs. Satisficing theory seems like a good starting point for this adaptation. ${ }^{12,17}$

For the modified connectivity robustness metric, research looking into allowing more robust connections is needed. The proposed metric only ensures one path from a node to the base station. If nodes were faulty, ensuring multiple paths to information neighborhood members would provide a more robust solution.

\section{Acknowledgments}

This research was supported by the National Science Foundation under Information Technology Research Grant CCF-0428004.

\section{References}

${ }^{1}$ Zhan, P., Casbeer, D., and Swindlehurst, A., "A Centralized Control Algorithm for Target Tracking with UAVs," Proc. 39th Asilomar Conf. Signals, Systems, and Computers, Pacific Grove, CA, November 2005.

${ }^{2}$ Jadbabaie, A., Lin, J., and Morse, A., "Coordination of groups of mobile autonomous agents using nearest neighbor rules," Automatic Control, IEEE Transactions on, Vol. 48, No. 6, 2003, pp. 988-1001.

${ }^{3} \mathrm{Fax}$, J. and Murray, R., "Information flow and cooperative control of vehicle formations," Automatic Control, IEEE Transactions on, Vol. 49, No. 9, 2004, pp. 1465-1476.

${ }^{4} \mathrm{Kim}, \mathrm{Y}$. and Mesbahi, M., "On maximizing the second smallest eigenvalue of a state-dependent graph Laplacian," Proceedings of the American Control Conference, 2005, pp. 99-103.

${ }^{5}$ Zavlanos, M. and Pappas, G., "Controlling Connectivity of Dynamic Graphs," Decision and Control, 2005 and 2005 European Control Conference. CDC-ECC'05. 44th IEEE Conference on, 2005, pp. 6388-6393.

${ }^{6}$ Qiu, Q. and Kamal, A., "Coverage and connectivity control of wireless sensor networks under mobility," High Performance Switching and Routing, 2005. HPSR. 2005 Workshop on, 2005, pp. 177-181.

${ }^{7}$ Spanos, D. P. and Murray, R. M., "Robust Connectivity of Networked Vehicles," Proceedings of the IEEE Conference on Decision and Control, 2004.

${ }^{8}$ Spanos, D. P. and Murray, R. M., "Motion Planning with Wireless Network Constraints," Proceedings of the American Control Conference, 2005.

${ }^{9}$ Wang, X., Xing, G., Zhang, Y., Lu, C., Pless, R., and Gill, C., "Integrated coverage and connectivity configuration in wireless sensor networks," Proceedings of the 1st international conference on Embedded networked sensor systems, 2003, pp. 28-39.

${ }^{10}$ Zou, Y. and Chakrabarty, K., "A distributed coverage-and connectivity-centric technique for selecting active nodes in wireless sensor networks," Computers, IEEE Transactions on, Vol. 54, No. 8, 2005, pp. 978-991.

${ }^{11}$ Liu, J., Reich, J., and Zhao, F., "Collaborative In-Network Processing for Target Tracking," EURASIP Journal on Applied Signal Processing, Vol. 4, 2003, pp. 378-391.

${ }^{12}$ Hill, J., Nokleby, M., Archibald, J., Frost, R., and Stirling, W., "Cooperative graph search by a system of autonomous agents," Systems, Man and Cybernetics, 2005 IEEE International Conference on, Vol. 1, 2005, pp. 121- 126.

${ }^{13}$ Keller, D., Moon, T., and Gunther, J., "Source Localization from Moving Arrays of Sensors," Proc. 39th Asilomar Conf. Signals, Systems, and Computers, Pacific Grove, CA, November 2005.

${ }^{14}$ Casbeer, D. W., Zhan, P., and Swindlehurst, A. L., "A Non-Search Optimal Control Solution for a Team of MUAVs in a Reconnaissance Mission," Proc. of the IEEE Int. Conf. on Acoustic, Speech and Signal Processing, 2006.

${ }^{15}$ Beard, R. W., Kingston, D., Quigley, M., Snyder, D., Christiansen, R., Johnson, W., McLain, T., and Goodrich, M., "Autonomous Vehicle Technologies for Small Fixed Wing UAVs," AIAA Journal of Aerospace Computing, Information, and Communication, Vol. 2, No. 1, 2005, pp. 92-108.

${ }^{16}$ Diestel, R., Graph Theory, Kluwer Academic Publishers, 2005.

${ }^{17}$ Stirling, W., Satisficing Games and Decision Making: With Applications to Engineering and Computer Science, Cambridge University Press, 2003. 


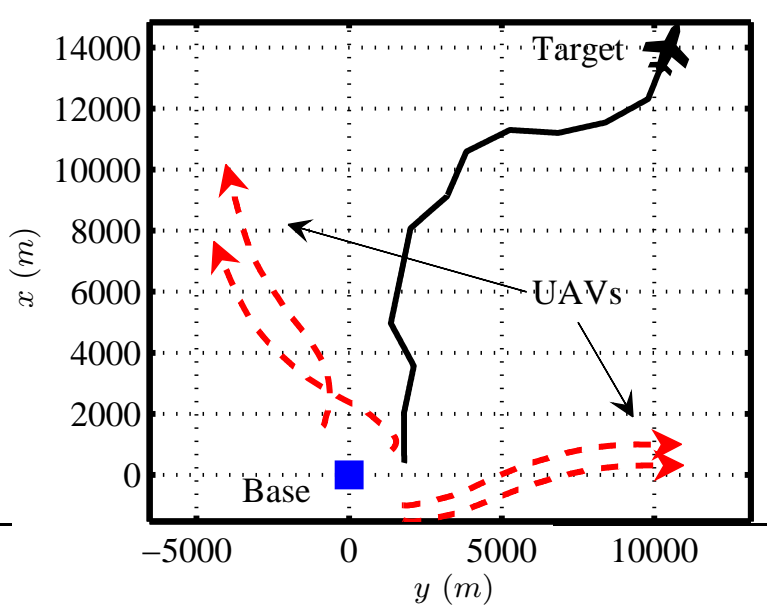

(a) Controlled: $\Psi_{\text {opt }, 1}$

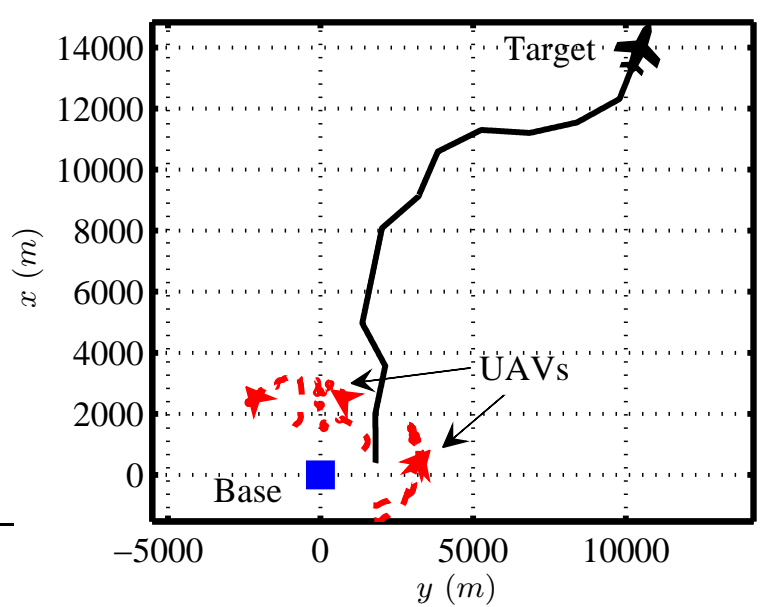

(b) Connectivity Robustness: $\Psi_{\text {opt }, 2}$

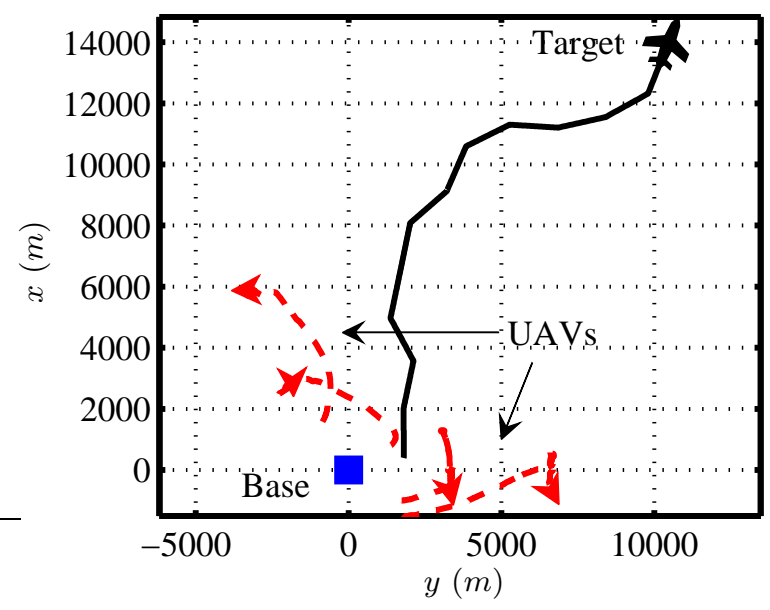

(c) Modified connectivity robustness, using the link neighborhood: $\Psi_{o p t, 3}$

Figure 4. Trajectory plots.

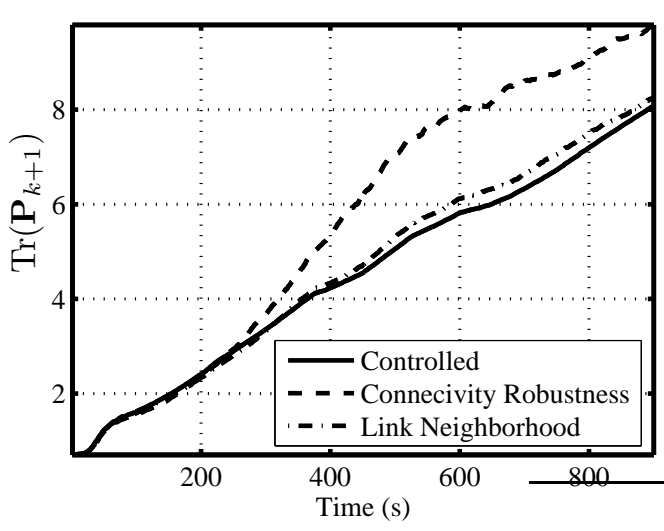

(a) Cost plot

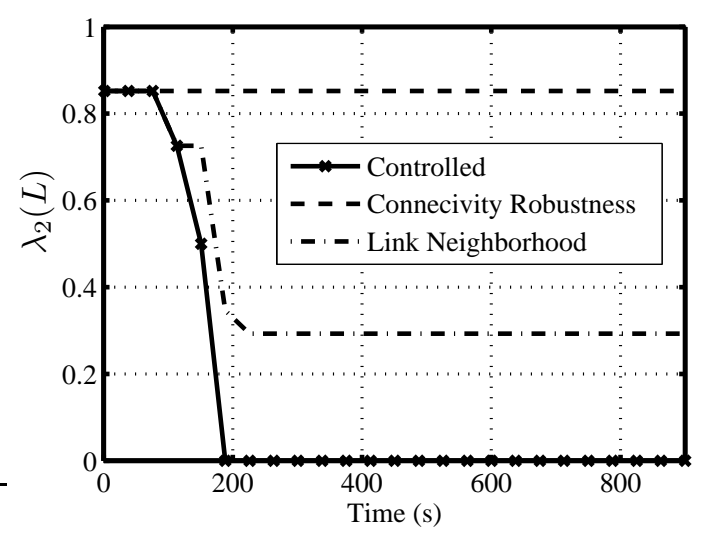

(b) Second eigenvalue of the network graph's Laplacian matrix

Figure 5. Cost plots. 